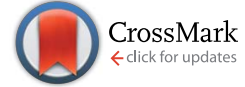

Cite this: RSC Adv., 2014, 4, 57173

\title{
Bacterial nanocellulose with a shape-memory effect as potential drug delivery system $\uparrow$
}

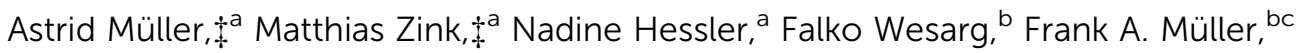 \\ Dana Kralisch ${ }^{\mathrm{ac}}$ and Dagmar Fischer*ac
}

\begin{abstract}
In the steadily emerging field of applications for the natural biopolymer bacterial nanocellulose (BNC), the development of environmentally-friendly and cost-saving techniques to form xerogels by partial or complete dewatering is of great interest for convenient storage, handling and a reduced risk of microbial contamination. Air-dried BNC itself is not able to rehydrate after complete drying due to a structural collapse. In the present paper, it was supplemented with different hydrophilic, water-binding additives and characterized regarding morphology, re-swelling behavior, mechanical stability and potential as drug delivery system. A fast rehydration could be obtained by the addition of magnesium chloride $>$ glucose > sucrose $>$ sorbitol, with a maximum re-swelling percentage up to about $88 \%$ (magnesium chloride) of the initial wet weight. In contrast, poly(ethylene glycol) $(8 \mathrm{kDa})$, lactose and trehalose reached only 12$30 \%$ whereas mannitol and sodium chloride only had a negligible effect. A high re-swelling value was found to be correlated with the preservation of the three-dimensional BNC network structure and mechanical characteristics such as compression and tensile strength. Confirming the relevance of these findings, the use of the hydrophilic model drug azorubine demonstrated the applicability of the shapememorized bacterial nanocellulose as drug delivery system with controllable release profiles.
\end{abstract}

Received 5th September 2014 Accepted 24th October 2014

DOI: $10.1039 / c 4 r a 09898 f$

www.rsc.org/advances well as drug delivery systems. ${ }^{2-4}$ An exceptionally high mechanical and thermal stability, high water binding capacity in combination with a high purity and biocompatibility distinguish BNC from other natural hydrogels in the field., Furthermore, a wide-ranging variety of modifications in situ during the synthesis of BNC as well as after formation of the material (post synthesis) accomplish a broad spectrum of BNC materials with different structural and physicochemical characteristics customizable for each desired application. ${ }^{7-9}$

For applications in tissue engineering, moist wound treatment, or as blood-vessel substitute, the high-water-content hydrogel is preferred. However, from the viewpoint of material storage and packaging, convenient handling and a reduced risk of microbial contamination, a partial or complete dewatering to form xerogels is desired. The ability of BNC to absorb or provide fluid can be adjusted by full or partial dewatering. Several techniques for the dewatering of BNC were described in the literature: (i) freeze-drying by sublimation of frozen water for the production of aerogels, (ii) critical point drying by treatment with supercritical carbon dioxide after replacement of water with suitable liquid solvents, (iii) stepwise solvent exchange for water displacement, (iv) water removal under pressure using water-absorbing materials and/or additional heating to prepare thin foils and membranes, and (v) air-drying by evaporation of water at elevated temperatures or vacuum.

Freeze-drying was described as a gentle drying technique forming dried BNC materials, with only a slightly reduced water 
content up to $70 \%$ of the original water content after reswelling. However, structural changes such as partial aggregation of free polymer strands were observed. ${ }^{10}$ In drug delivery applications, uptake of proteins into the gel was found to be lower for freeze-dried compared to never-dried BNC related to changes of the fiber network during lyophilization. ${ }^{11}$

Drying with supercritical carbon dioxide at $40{ }^{\circ} \mathrm{C}$ and $100 \mathrm{bar}$ after quantitative solvent exchange resulted in structural changes characterized by a macroporous system and clew-type agglomeration of the cellulose ribbons compared to the native network. ${ }^{12}$ Maeda et al. ${ }^{13}$ applied supercritical drying with ethanol under rough conditions at $243{ }^{\circ} \mathrm{C}$ and $6.38 \mathrm{MPa}$ so that the material underlies structural modifications due to dehydratization reactions and hornification. Additionally, this type of drying technique requires usually organic solvents which might have a critical impact on human hazard, environment and possibly loaded drugs. Hot-pressing was found to result in a collapse of the BNC network structure, densification and a decrease of the sample thickness. ${ }^{14}$ As major disadvantages, all these techniques are cost and time-consuming, require expensive equipment and produce in some cases solvent waste critical for human health and environment. Additionally, taking such dried BNC fleeces as drug delivery systems into consideration, all of these methods suffer from high thermal and mechanical stresses for the loaded drugs and excipients, preventing their use in various pharmaceutical applications.

Air-drying of BNC was described to result in a complete loss of the three-dimensional network structure, intense wrinkling as a result of strong shrinkage, superficial hornification, fibre aggregation and conclusively, a reduction of the number and sizes of pores. ${ }^{15-17}$ As a result of these changes, collapsed membranes with strongly reduced re-swelling properties and the almost entire loss of the water holding capacity were observed. ${ }^{18,19}$

In the present paper a cost effective and environmentally friendly route to overcome these issues and to reproducibly produce air-dried BNC with a high re-swelling potential is presented. Several hydrophilic, moisture-binding excipients such as sugars, inorganic salts, polyalcohols, and polymers were used as additives to accomplish a controlled water uptake into airdried BNC fleeces. Important properties of these modified materials are reported with regard to morphology, swelling properties, mechanical stability and drug release behavior in comparison to unmodified air-dried and freeze-dried BNC.

\section{Experimental}

\section{Preparation and characterization of bacterial nanocellulose}

Bacterial nanocellulose was produced by static cultivation of Komagataeibacter xylinus (formerly called Gluconacetobacter xylinus) strain DSM 14666 (culture collection of the FriedrichSchiller-University Jena, deposited at the German Collection of Microorganisms and Cell Cultures, Braunschweig, Germany) in 24-well plates as described previously. ${ }^{8}$ Fleeces were purified by treatment with $0.1 \mathrm{~N}$ aqueous sodium hydroxide solution and neutralized by rinsing with deionized water followed by autoclaving at $121^{\circ} \mathrm{C}$ for $20 \mathrm{~min}$ and 2 bar. Purified wet BNC material was referred to as "never-dried" state in the following, whereas dried BNC fleeces were either named "air-dried" or "freeze-dried" depending on whether air-drying at $22{ }^{\circ} \mathrm{C}$ or lyophilisation (63 Pa, $-85{ }^{\circ} \mathrm{C}$, ALPHA 2-4 LSC, Martin Christ, Osterode, Germany) were applied. Characterization of the BNC was performed by examination of different sample parameters (weight, thickness, diameter, volume) and the macroscopic morphology as described before. ${ }^{11}$

\section{Additives and additive solutions}

The following additives were selected as rehydrating agents: (i) the carbohydrates glucose, saccharose, lactose monohydrate and trehalose dihydrate, (ii) the sugar alcohols sorbitol and mannitol, (iii) the polymer $8 \mathrm{kDa}$ poly(ethylene glycol) and (iv) the inorganic salts sodium chloride and magnesium chloride hexahydrate. All substances were purchased from Carl Roth GmbH \& Co. KG, Karlsruhe, Germany, in analytical grade and used without further purification. For preparation of the $10 \%$ (w/v) additive solutions double distilled water was used.

\section{Supplementation with additives, air-drying and re-swelling of BNC}

For supplementation never-dried BNC samples were immersed each in $10.0 \mathrm{~mL} 10 \%(\mathrm{w} / \mathrm{v})$ aqueous additive solution. Incubation was performed on an orbital shaker (70 rpm, POS-300 orbital shaker, Grant Instruments, Cambridge, UK) for $24 \mathrm{~h}$ at $22{ }^{\circ} \mathrm{C}$ in closed vials. After $24 \mathrm{~h}$ loading, BNC fleeces were removed from the loading solution and characterized regarding morphology and dimensions. ${ }^{11}$ Afterwards, the loaded samples were air-dried at room temperature for $96 \mathrm{~h}$ placed on filter paper. Re-swelling was performed under shaking in $20.0 \mathrm{~mL}$ double distilled water per fleece in closed vials $\left(22^{\circ} \mathrm{C}, 70 \mathrm{rpm}\right.$, POS-300 orbital shaker) up to $168 \mathrm{~h}$. The influence of drying and rehydration on the BNC sample characteristics was investigated by determination of the fleece parameters at selected time points. Sample re-swelling $\left(R_{\mathrm{S}}\right)$ in percentage was calculated based on the following formula:

$$
R_{\mathrm{s}}=\frac{w_{\mathrm{s}}}{w_{\mathrm{i}}} \times 100
$$

where $w_{\mathrm{s}}$ represents the sample weight in the swollen state at certain time points of rehydration and $w_{\mathrm{i}}$ the initial weight before the drying step. Changes of the sample weight after incubation with additives were indicated as percentage of initial sample weight before supplementation of never-dried BNC with additives, whereas for quantification of the drying and the rehydration process, weight changes were calculated as percentage of sample weight after additive loading. Furthermore, the macroscopic appearance of fleeces was visually inspected by taking photographs of the samples after additive loading as well as after completion of drying and rehydration step in macro-mode (Digital camera FinePix E550, Fujifilm Corporation, Tokyo, Japan). Negative controls consisting of BNC fleeces that were incubated only in double distilled water without any additive and treated afterwards by air-drying or freeze-drying were mentioned as air-dried and freeze-dried 
controls, respectively, in the following. All parameters were calculated based on determinations of three samples for each additive.

\section{Determination of water content}

The residual water content of dried samples was determined by the Karl-Fischer titration method $^{20}$ calculating the amount of titrated water from the quantity of current necessary to produce iodine in a coulometric cell with diaphragm (analyst solution Hydranal®-Coulamat A, catalyst solution Hydranal®-Coulamat CG, Fluka Analytical, St. Gallen, Switzerland; Karl-FischerTitrator Aqua 3000, ECH, Halle, Germany). Based on the measured water content and dry weight of the additive loaded samples as well as air-dried controls, the amount of additive in the dried BNC was calculated (mean $\pm \mathrm{SD}, n=4$ ).

\section{Fehling's test}

To visualize the distribution of sugars in the BNC hydrogel matrix $10 \%(\mathrm{w} / \mathrm{v})$ carbohydrate (glucose, sucrose) loaded samples were treated each with $2.0 \mathrm{~mL}$ Fehling's reagent consisting of a $1: 1$ mixture of Fehling I (6.9\% (w/v) copper(II) sulphate pentahydrate, Merck KGaA, Darmstadt, Germany) and Fehling II (34.6\% (w/v) potassium sodium tartrate tetrahydrate, Merck KGaA, Darmstadt, Germany; $10.0 \%$ (w/v) sodium hydroxide, Carl Roth) aqueous solutions ${ }^{21}$ at $70 \mathrm{rpm}$ (POS-300 orbital shaker) for $20 \mathrm{~min}$ followed by heating to boiling. Never-dried controls were treated in the same way for comparison. The assay is based on the reducing effect of monosaccharide's converting copper(II) ions to the red coloured precipitate of copper(I) oxide as an indicator for the presence of reducing sugars. Samples loaded with sucrose were additionally treated with $2.0 \mathrm{~mL} 1 \mathrm{~N}$ hydrochloric acid (VWR, Darmstadt, Germany) to degrade disaccharides to monosaccharides before treatment with Fehling's reagent. Staining of samples was performed immediately after loading and rehydration $(n=3)$. Photographs (Digital camera FinePix E550, Fujifilm Corporation, Tokyo, Japan) of cross-sections were taken in macro-mode.

\section{Scanning electron microscopy (SEM)}

Rehydrated BNC samples supplemented with glucose, sucrose and magnesium chloride were lyophilized and sputter coated with gold at $15 \mathrm{~mA}$ in argon atmosphere (S150B, Edwards, Crawley, WestSussex, UK). The micrographs of the crosssections, the top and the bottom surfaces of the samples were recorded in vacuo at an acceleration voltage of $20 \mathrm{kV}$ (S440i, Leica Microsystems $\mathrm{GmbH}$, Wetzlar, Germany) in comparison to never-dried and rehydrated air-dried controls.

\section{Mechanical characterization}

Compression tests were performed using rehydrated air-dried 12-well format BNC samples that were treated with the selected additives glucose, sucrose and magnesium chloride as described above. The tests were carried out using the Universal testing machine TIRAtest 2710 (Tira GmbH, Schalkau, Germany) connected to a $1 \mathrm{kN}$ load cell. The samples were compressed perpendicularly to the BNC surface at a deformation rate of $10 \mathrm{~mm} \mathrm{m^{-1 }}$ until a compression of $50 \%$ was reached. The nominal compression strength $\left(\sigma_{\mathrm{C}}\right)$ was calculated. Additional tensile tests were performed using re-swollen dumbbell-shaped samples, which were treated with the additives in the same way as the 12-well formatted samples. All samples were mounted in sample grips and stretched at a deformation rate of $50 \mathrm{~mm} \min ^{-1}$ up to the point of failure (TIRAtest 2710 , load cell of $0.1 \mathrm{kN})$. The stress $\left(\sigma_{\mathrm{T}}\right)-\operatorname{strain}\left(\varepsilon_{\mathrm{T}}\right)$ curves were recorded and the ultimate tensile strength $\left(\sigma_{\mathrm{TS}}\right)$ was determined. ${ }^{22}$ Mechanical properties were analyzed in comparison to the behavior of never-dried as well as rehydrated freeze-dried and air-dried controls. Mean values and standard deviations were based on at least four determinations of individual samples for each sample type.

\section{Loading and release of a hydrophilic dye as model drug}

To investigate the influence of the additive supplementation on drug loading and release to and from BNC samples the red dye azorubine (Sicovit Azorubine 85, E 122, BASF Chem Trade $\mathrm{GmbH}$, Burgbernheim, Germany) was selected as hydrophilic model drug. The loading step was performed by incubation of the never-dried samples in $10.0 \mathrm{~mL}$ aqueous solution of $10 \%(\mathrm{w} / \mathrm{v})$ glucose containing $0.5 \mathrm{mg} \mathrm{mL}^{-1}$ azorubine followed by $96 \mathrm{~h}$ airdrying prior release (air-dried glucose). Samples incubated in dye solution without the addition of sugar were used as controls. Both, loading and release were performed at $22{ }^{\circ} \mathrm{C}$ under shaking (loading: $70 \mathrm{rpm}$, release: $200 \mathrm{rpm}$, POS-300 orbital shaker). For release samples were transferred to 20.0 $\mathrm{mL}$ double distilled water as release medium after air-drying (air-dried glucose, air-dried control) or directly after the loading step for never-dried controls. A second release step was performed by transfer of each sample to $20.0 \mathrm{~mL}$ fresh double distilled water after $168 \mathrm{~h}$ of the first release. Aliquots of the supernatants of loading and release media were collected at specified time intervals. Azorubine quantification was carried out spectrophotometrically at $515 \mathrm{~nm}$ (Beckmann DU 640 spectrophotometer, Beckmann Instruments Inc., Fullerton, USA) using a calibration curve. The difference between the initial amount of azorubine in the loading solution and the amount of azorubine in the supernatant after loading was calculated for determination of the loaded amount of dye and the uptake capacity (loaded azorubine as percentage of initial dye amount in the loading solution) as described before. ${ }^{11}$ In parallel, the release of glucose was measured reflectometrically (Reflectoquant Analysis System, Merck, Darmstadt, Germany) as described before ${ }^{9}$ at selected time intervals. All experiments were run in triplicate and were repeated once.

\section{Results}

\section{Preparation of re-swellable BNC}

Bacterial nanocellulose was produced by Komagataeibacter xylinus at the air-liquid interface as stable hydrogel fleeces. Purified never-dried hydrogels were characterized by a disk shape and a macroscopically intact morphology. Produced 
under the selected conditions in 24-well plates, never-dried BNC fleeces showed highly reproducible sample parameters such as a mean weight of about $1.2 \mathrm{~g} \pm 0.1 \mathrm{~g}$. Their diameter $(1.6 \mathrm{~cm} \pm$ $0.1 \mathrm{~cm}$ ) was predetermined by the dimensions of the cell culture wells, and sample thickness ranged from 0.6 to $0.7 \mathrm{~cm}$. The volume was calculated as $1.4 \mathrm{~mL} \pm 0.2 \mathrm{~mL}$.

For the production of air-dried BNC with an enhanced swelling rate, a post synthesis modification was applied comprising a $24 \mathrm{~h}$ incubation of native, wet BNC in an additive solution and a subsequent thorough air-drying to constant mass at room temperature. As additives, several chemically different substances with hydrophilic characteristics, well known osmotic effects, high hygroscopicity and polar moieties were selected: (i) carbohydrates, (ii) polyols, (iii) a nonionic polymer, and (iv) inorganic salts.

Based on preliminary experiments with different loading times and additive concentrations (data not shown), optimized conditions for loading of native $\mathrm{BNC}$ in $10 \%(\mathrm{w} / \mathrm{v})$ additive solutions over $24 \mathrm{~h}$ submersed under shaking at room temperature were selected for the following experiments. Fig. 1 shows the changes of BNC sample weight after $24 \mathrm{~h}$ loading compared to the starting weight which was set as $100 \%$. All samples treated with an additive demonstrated a slightly higher wet weight after loading compared to the starting weight and the negative air-dried and freeze-dried controls. The highest increase in weight could be achieved by the treatment with the inorganic salts magnesium chloride $(106.2 \% \pm 0.8 \%)$ and sodium chloride $(106.9 \% \pm 3.4 \%)$ followed by the sugars sucrose and lactose $(105.5 \% \pm 1.4 \%$ and $105.0 \% \pm 2.2 \%$, respectively) as well as sorbitol $(104.1 \% \pm 1.1 \%)$ as additives. All other samples showed lower weight changes $\leq 3 \%$. After $24 \mathrm{~h}$ loading with the different additives, the wet BNC samples were air-dried at room temperature for $96 \mathrm{~h}$, representing the time frame to reach a constant mass for all additive loaded samples. Fig. 1 shows the corresponding dry weight of the samples as percentage of the sample weight before drying. The highest dry weights were obtained by supplementation of BNC with magnesium chloride $(12.2 \% \pm 0.3 \%)$, followed by sucrose, glucose and sorbitol $(5.8 \% \pm 0.8-5.7 \% \pm 0.2 \%)$ as well as $8 \mathrm{kDa}$ PEG $(4.4 \% \pm 0.3 \%)$ and trehalose $(4.0 \% \pm 0.4 \%)$. For all other

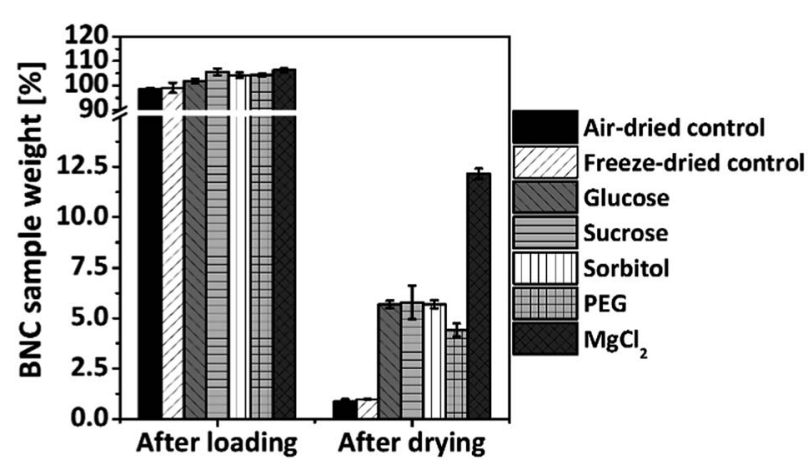

Fig. 1 Representative sample weights of BNC determined after $24 \mathrm{~h}$ loading in 10\% aqueous additive solution and after $96 \mathrm{~h}$ air-drying compared to air-dried and freeze-dried controls (mean $\pm \mathrm{SD}, n=3$ ). additives dry weights $\leq 2.6 \%$ could be observed. In comparison, the values for air-dried and freeze-dried controls ranged about $0.9-1.0 \%$.

\section{Swelling dynamics of additive loaded BNC}

Air-dried samples were allowed to rehydrate in double distilled water for $168 \mathrm{~h}$ to examine their re-swelling properties. Fig. 2A shows the time-dependent re-swelling $\left(R_{\mathrm{S}}\right)$ of the air-dried additive loaded BNC samples in comparison to air-dried and freeze-dried controls. The investigation of the extent and speed of rehydration revealed major differences between the fleeces dependent on the type of additive. The time-dependent swelling curves showed an exponential profile with a steep rise in the curves in the first 4 hours, followed by a slower increase and/or reaching steady state after 24 to 96 hours. This was true for all additive loaded BNCs with exception of lactose, trehalose, mannitol, and sodium chloride. Lactose and trehalose induced only a limited re-swelling up to $8.1 \%$ and $6.0 \%$, respectively, in the initial 4 hours without significant changes in the following hours. Mannitol and sodium chloride did only have a negligible effect on the re-swelling behavior without any shape memorizing effect, similar to air-dried controls.

Magnesium chloride was found to be the most effective reswelling additive that induced not only the fastest but also the highest re-swelling $(88.4 \% \pm 1.5 \%)$. It reached values that were comparable to the freeze-dried controls $(86.3 \% \pm 1.5 \%)$. Glucose $(64.8 \% \pm 10.8 \%)$, sucrose $(56.1 \% \pm 11.3 \%)$, sorbitol $(50.2 \% \pm 12.1 \%)$ and $8 \mathrm{kDa}$ PEG $(30.0 \% \pm 2.4 \%)$ enhanced the water uptake into the air-dried BNC with decreasing efficiency. The faster the water uptake during the first $4 \mathrm{~h}$, the more effective was the additive as shape-memorizing agent.

\section{Residual water content and additive amount of air-dried BNC}

To examine the impact on the re-swelling behavior of supplemented BNC, residual water and additive amount of the airdried samples were determined by Karl-Fischer titration and compared to air-dried and freeze-dried controls (Fig. 2B and C). With exception of magnesium chloride loaded BNC $(61.8 \% \pm$ $0.9 \%$ ), the residual water content was found for all samples to be in the range from $0.5 \%$ to $6.5 \%$ of the dry weight with a slight trend to higher values for sugar loaded BNC (Fig. 2B). The values were comparable to air-dried and freeze-dried controls and already published data for BNC. ${ }^{15}$

Based on the measured dry weights and the water content (Fig. 1, ESI 1 and $2 \dagger$ ), the amount of additive incorporated into the dried BNC was calculated. From the correlation of these parameters with the re-swelling data (Fig. 2C), it could be concluded that a higher dry weight was mainly related to a higher additive amount in the dried sample and not to a higher water content. The only exception was BNC supplemented with magnesium chloride that was characterized by an exceptionally high water content as described above.

Taken all these data together, this allows the classification of the tested additives in four groups: (i) magnesium chloride as the most effective re-swelling agent characterized by a high residual water content, (ii) glucose, sucrose and sorbitol with 

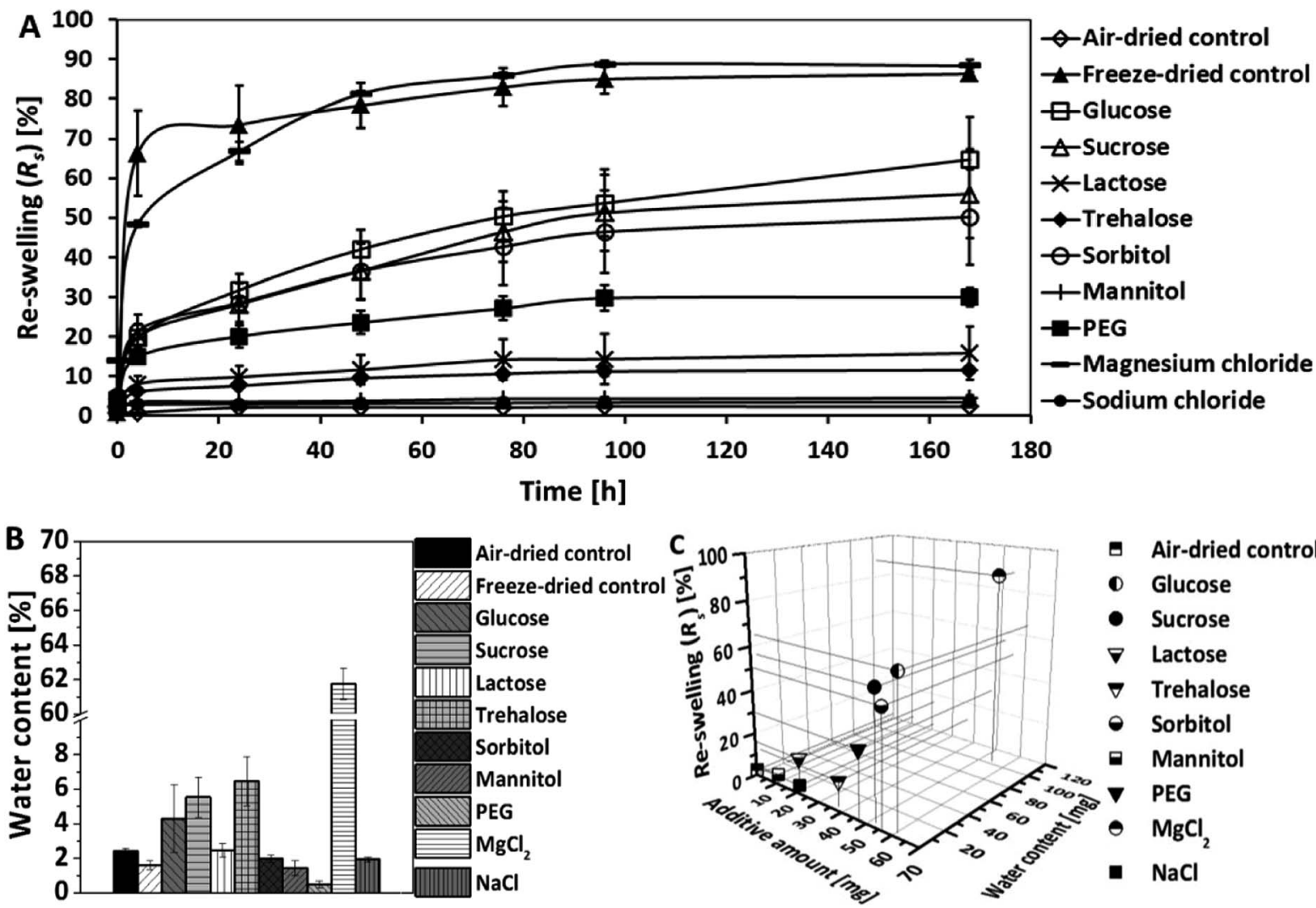

Fig. 2 A Time-dependent re-swelling $\left(R_{\mathrm{s}}\right)$ of additive loaded and air-dried BNC in comparison to air-dried and freeze-dried controls (mean $\pm \mathrm{SD}$, $n=3)$. B Residual water content of air-dried BNC samples determined by Karl Fischer titration compared to air-dried and freeze-dried controls. Mean values and standard deviation in percentage of sample dry weight were calculated based on four determinations for each tested additive. $C$ Correlation of BNC re-swelling $\left(R_{\mathrm{s}}\right)$ after 168 hours, residual water content and additive amount of air-dried BNC samples.

the highest remaining amount of additive that accomplish an enhanced water uptake, (iii) PEG > lactose, trehalose showing only limited re-swelling that is correlated with lower amounts of additives, and (iv) sodium chloride as well as mannitol without relevant re-swelling characteristics and low residual additives in the fleeces.

\section{Distribution of additives in the BNC hydrogels}

After $24 \mathrm{~h}$ loading and $168 \mathrm{~h}$ re-swelling, distribution of the additives in BNC fleeces loaded with glucose and sucrose were analyzed by Fehling's test (Fig. 3). Both carbohydrates were selected as representatives because of their ability to enhance the swelling properties of BNC and their simple analysis for detection. After $24 \mathrm{~h}$ incubation, never-dried controls were stained homogeneously blue by unreacted Fehling's reagent, indicating that the reagent could reach all parts of the hydrogel. After loading of BNC samples with the sugars, they were homogeneously stained red-brown demonstrating a uniform carbohydrate distribution. After $168 \mathrm{~h}$ re-swelling, sugar loaded samples showed only a faint red color mainly located in the core of the gels, suggesting that during the re-swelling process the sugar was removed from outside to inside. Additive molecules localized in the sample core had to overcome longer diffusion distances from the core to the surrounding medium and therefore remained visible in the sample core after re-swelling.

\section{Morphological and structural investigations}

The quantitative measurements during and after loading, airdrying and re-swelling of BNC were completed by the
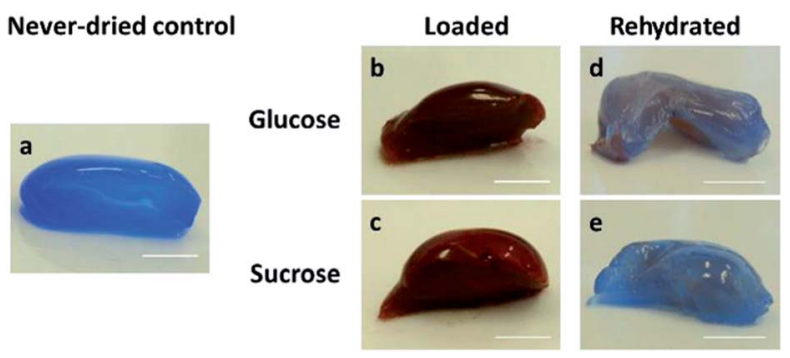

Fig. 3 Distribution of glucose and sucrose in the BNC gels after staining by Fehling's reagent (scale bars $5 \mathrm{~mm}$ ). The never-dried, carbohydrate-free BNC control (a) itself did not reduce the blue Fehling's reagent. Immediately after loading with glucose (b) or sucrose (c) the materials demonstrated a homogeneous brown coloring suggesting uniform sugar distribution. After air-drying and rehydration ( $\mathrm{d}$ and $\mathrm{e}$ ) the brown coloring was reduced demonstrating the release of the carbohydrates during the rehydration process (scale bars, $5 \mathrm{~mm}$ ). 
macroscopic inspection of sample morphologies (Fig. 4) and sample dimensions represented by changes of the sample volume (Fig. 5 and ESI $3 \uparrow$ ). To be able to directly compare the sizes of the BNC fleeces, a scale bar of $5 \mathrm{~mm}$ was inserted into each photograph since they were taken with varying distances between camera and fleece (Fig. 4).

Directly after loading, none of the BNC hydrogels showed any alteration of shape, dimensions or morphology indicating that they were stable during this process. Freeze-dried controls with a high dimensional stability formed aerogels that were characterized by an irregular surface but nearly the same cylindric form than never-dried BNC. During air-drying all BNC hydrogels evaporated almost completely and became thin, collapsed, light weight specimens with transparent or matt white surfaces and a hard (e.g. air-dried control, sodium chloride loaded samples) or flexible texture (e.g. magnesium chloride loaded samples). They underwent a dramatic change of

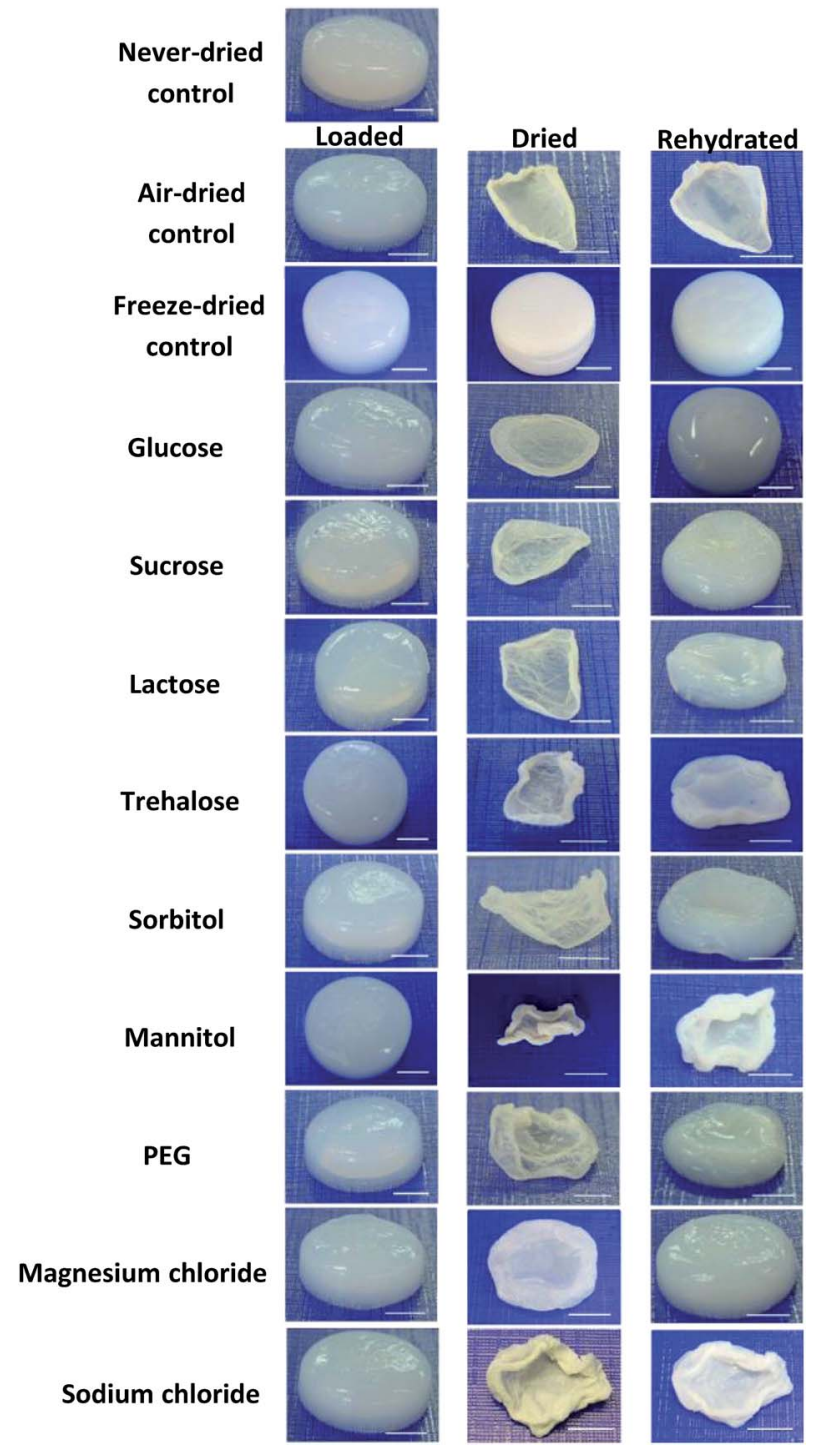

Fig. 4 Macroscopic BNC sample morphology after $24 \mathrm{~h}$ additive loading, $96 \mathrm{~h}$ air-drying and $168 \mathrm{~h}$ rehydration (scale bars, $5 \mathrm{~mm}$ ).

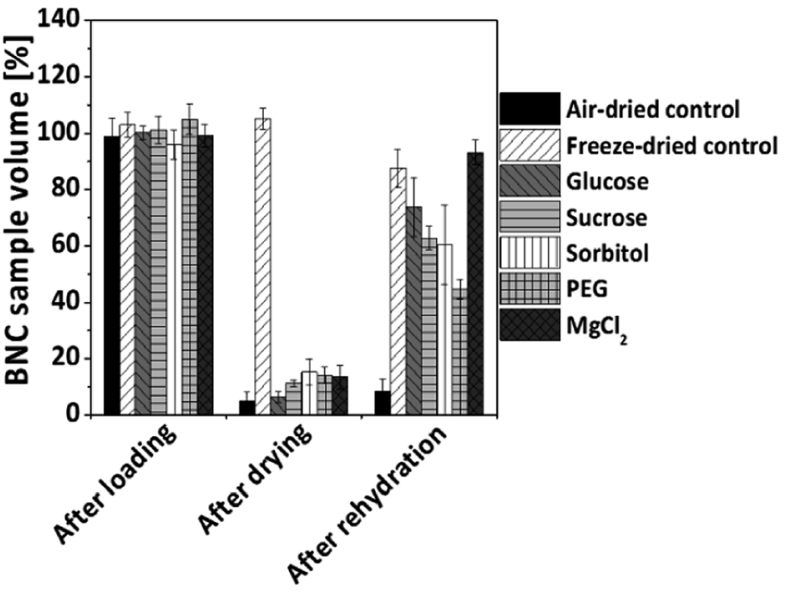

Fig. 5 Additive loaded BNC with high re-swelling capacity: comparison of BNC sample volume based on measured sample dimensions (thickness, diameter) and given as percentage of initial volume of additive loaded, dried and re-swollen samples compared to air-dried and freeze-dried controls (mean $\pm \mathrm{SD}, n=3$ ).

their morphology by strong shrinking and in some cases intense wrinkling. The loaded wet BNC hydrogels with originally a homogeneous and smooth surface reduced their thickness from $0.6-0.7 \mathrm{~cm}$ to $0.1-0.3 \mathrm{~cm}$. The decrease in sample weight described above was therefore accompanied by a drastic decrease in sample thickness and volume during the drying process.

Morphologically, air-dried controls did not show any signs of water uptake or re-swelling after transfer into water at room temperature. Freeze-dried controls were able to absorb water, but were characterized by a rougher and more irregular surface compared to never-dried controls, suggesting moderate changes of the BNC network after drying and rehydration. Depending on the type of loaded additive, the BNC samples demonstrated different re-swelling properties and morphologies. Air-dried BNC loaded with magnesium chloride, glucose, sucrose, and sorbitol impressed by shape memory properties, showing after re-swelling nearly the same macroscopic morphology as the native material before treatment.

This was confirmed by their volumes after rehydration ranging from $93.2 \% \pm 4.3 \%$ (magnesium chloride) to $60.4 \% \pm$ $14.1 \%$ (sorbitol) compared to their volumes before the drying step. PEG, lactose, and trehalose loaded samples demonstrated an incomplete re-swelling with irregular surfaces and volumes between $44.6 \% \pm 3.6 \%$ (PEG) and $17.6 \% \pm 2.7 \%$ (trehalose) in comparison to their never-dried appearance. Inclusion of sodium chloride or mannitol into the BNC hydrogels resulted in sample volumes and morphologies comparable to the air-dried controls, showing nearly the same macroscopic morphology after rehydration than in the dried state. The morphological data correlated well with the results of the measurements of sample weight and re-swelling values during loading, air-drying and re-swelling steps described above.

Additionally, to investigate if changes of the threedimensional structure could be responsible for the differences 
of the re-swelling behavior, the network microstructure of the materials with enhanced water sorption was investigated by SEM after re-swelling in comparison to never-dried and rehydrated air-dried controls.

Top and bottom surfaces as well as cross-sections of these samples were microscopically investigated after lyophilization (Fig. 6). Never-dried controls (Fig. 6a-c) represented the typical BNC structure consisting of a dense top surface (Fig. 6a), a broad middle layer with a nanoscaled fiber network and a well defined pore system (Fig. 6b) as well as an open porous bottom surface formed by the pre-polymer (Fig. 6c). ${ }^{23}$ Corresponding to the macroscopic observations, the air-dried control (Fig. 6d-f) demonstrated a complete and irreversible loss of the threedimensional network structure due to aggregation of fibres and a reduction of the number of pores.

In contrast, the top and bottom surface of BNC samples with the most effective re-swelling (glucose, Fig. $6 \mathrm{~g}$ and i; sucrose, Fig. $6 \mathrm{j}$ and $\mathrm{l}$; magnesium chloride, Fig. $6 \mathrm{~m}$ and o) showed similar morphological characteristics and microstructures compared to the never-dried control (Fig. 6a and c). Additionally, glucose (Fig. 6h) and magnesium chloride (Fig. 6n) loaded samples retained an open porous network structure in the cross-sections almost equal to the never-dried control (Fig. 6b). BNC loaded with sucrose (Fig. 6k) demonstrated a partial densification of the fiber network with larger interstitial spaces which might be caused by parallel alignment of BNC fiber strands during air-drying and could be the explanation for the slightly lower re-swelling values compared to glucose loaded samples.

In conclusion, a high re-swelling of an additive loaded sample was found to correlate with a preservation of the threedimensional network structure. The irreversible collapse of the fiber network as observed for the air-dried control could be circumvented by the supplementation of BNC with selected additives leading to a reversible collapse during drying and advanced rehydration during re-swelling.
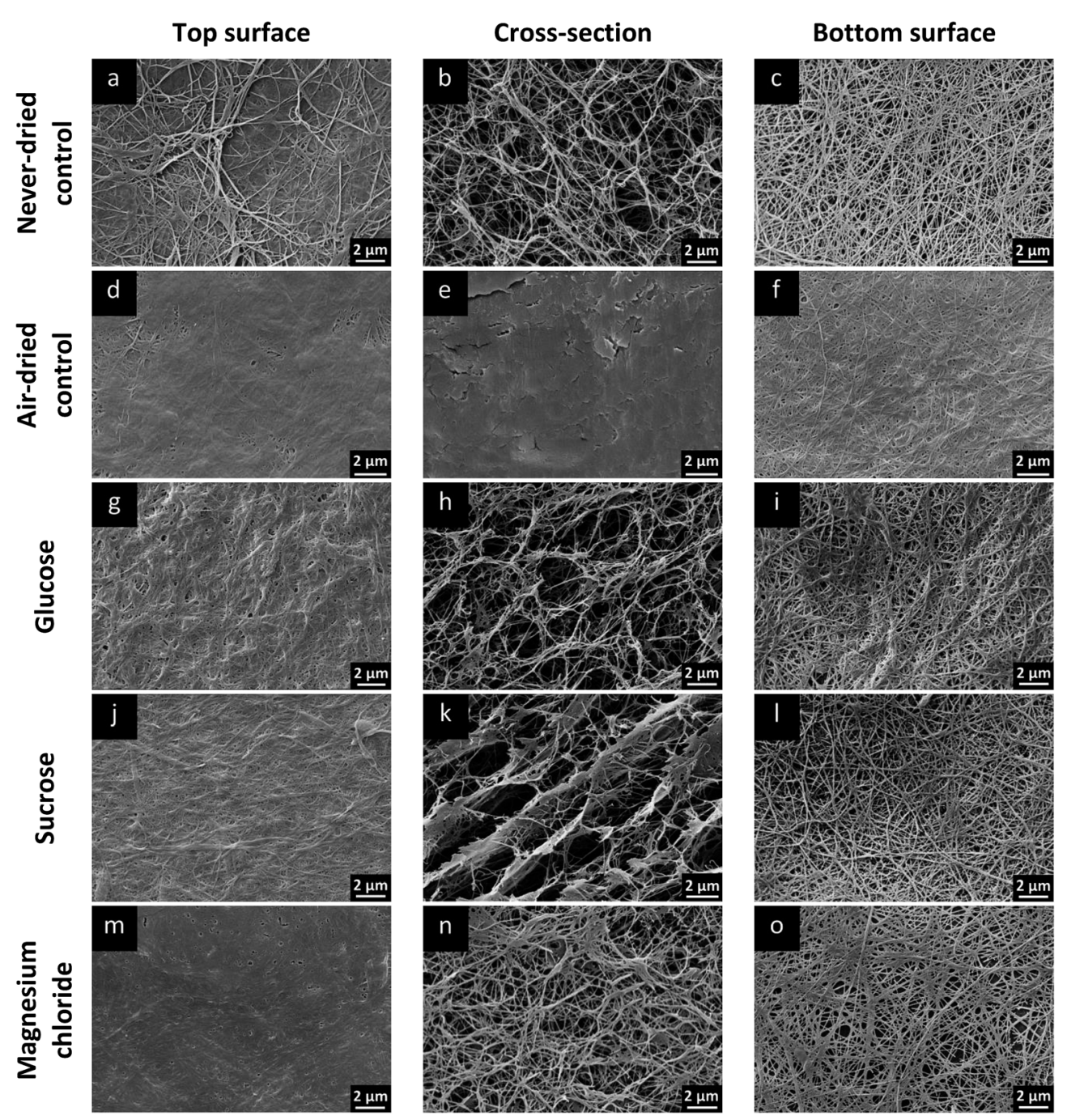

Fig. 6 SEM micrographs of the top surfaces ( $a, d, g, j$ and $m$ ), the cross-sections (b, e, h, k and $n$ ), and the bottom surfaces (c, $f, i, l$ and o) of neverdried $(a-c)$ and re-swollen air-dried $(d-f)$ controls compared to the additive loaded, air-dried samples after re-swelling [glucose, ( $g$-i); sucrose, $(\mathrm{j}-\mathrm{l})$; magnesium chloride, $(\mathrm{m}-\mathrm{o})]$ (scale bars, $2 \mu \mathrm{m})$. 


\section{Comparison of mechanical properties}

The influence of the additives on the mechanical properties of BNC was determined by compression and tensile tests (Fig. 7). Again, glucose, sucrose and magnesium chloride, were selected as additives. Nominal compression strength of rehydrated samples $\left(\sigma_{\mathrm{C}}\right)$ was found to be slightly increased by a factor of $1.2,1.4$, and 1.5 for magnesium chloride, glucose and sucrose loaded BNC, respectively, whereas the compression strength of re-swollen freeze-dried controls was strongly decreased by a factor of 2.5 compared to never-dried controls (Fig. 7A). Airdried controls could not be analyzed by compression tests due to their irreversible structural collapse.

Fig. 7B shows the stress $\left(\sigma_{\mathrm{T}}\right)$-strain $\left(\varepsilon_{\mathrm{T}}\right)$ curves of rehydrated additive loaded BNC compared to never-dried as well as reswollen freeze-dried and air-dried controls. The nominal tensile strength at maximum deformation force was determined from the curves and is shown in Fig. 7C. Additive loaded BNC samples behaved similar to never-dried, rehydrated airdried and freeze-dried controls with regard to the shape of the curves. Increasing development of strain resulted in an increase of tensile stress values until curve maxima representing nominal tensile strength at maximal deformation force were reached. Although an equal shape of the stress-strain curves with comparable tensile strain values in the range of 5 to $7 \%$ at maximal force was observed for all of the sample types (Fig. 7B), differences of the tensile strength values $\left(\sigma_{\mathrm{TS}}\right)$ of the samples were obtained (Fig. 7C).

Rehydrated supplemented BNCs showed tensile strength values in either the same (glucose: $0.392 \pm 0.037 \mathrm{MPa}$ ) or even moderate increased range (sucrose: $0.609 \pm 0.072 \mathrm{MPa}$, magnesium chloride: $0.539 \pm 0.030 \mathrm{MPa}$ ) compared to the never-dried controls $(0.373 \pm 0.033 \mathrm{MPa})$. Ultimate tensile strength of rehydrated freeze-dried and air-dried controls was found to be increased due to structural changes. Whereas tensile strength of the freeze-dried control was only increased by a factor of 2.4, air-dried controls showed a very strong increase by a factor of 69.1 compared to the never-dried control.

It is obvious from the compression and tensile tests that additive treatment influences the mechanical behavior of the BNC samples only slightly compared to the never-dried controls. Moderate increase of compression and tensile strength of the additive loaded samples might be related to slightly structural changes as well as residual amount of additives remaining in the sample core as it could be shown for the sugars by staining with Fehling's reagent. Rehydrated freezedried and air-dried controls showed differences compared to the native material characteristics due to irreversible structural changes during drying processes as also observed in the SEM investigations.

\section{Release of the hydrophilic dye azorubine}

The red dye azorubine was selected as low molar mass and hydrophilic model drug to investigate the applicability of shapememorized BNC as drug delivery system. Drugs with comparable physicochemical characteristics, that are relevant for medical applications of BNC could be e.g. polyhexanide, ${ }^{24}$
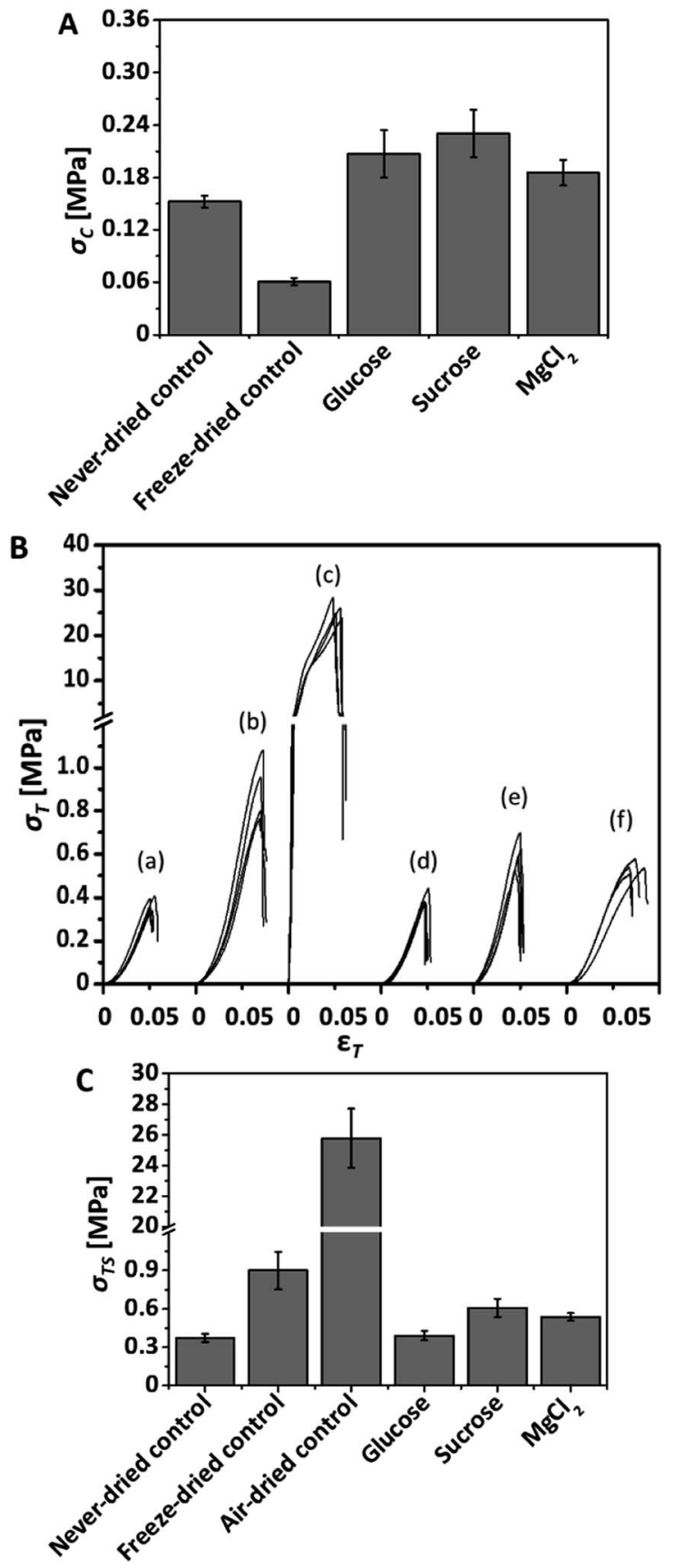

Fig. 7 A Compression strength $\left(\sigma_{C}\right)$ analysis of samples at $50 \%$ deformation and a deformation velocity of $10 \mathrm{~mm} \mathrm{~min}^{-1}$. Re-swollen samples loaded with glucose, sucrose, and magnesium chloride were analyzed in comparison to never-dried and re-swollen freeze-dried controls (mean $\pm \mathrm{SD}, n \geq 4$ ). B Tensile strength analysis of samples at $50 \mathrm{~mm} \mathrm{~min}^{-1}$ test velocity. Stress $\left(\sigma_{T}\right)$-strain $\left(\varepsilon_{T}\right)$ curves of never-dried (a), re-swollen freeze-dried (b) and air-dried (c) controls as well as reswollen samples loaded with glucose (d), sucrose (e), and magnesium chloride (f) are shown (four individual sample measurements for each investigated sample type). C nominal tensile strength ( $\left.\sigma_{\mathrm{TS}}\right)$ of samples at maximal deformation force. Tensile strength of re-swollen samples loaded with glucose, sucrose, and magnesium chloride in comparison to never-dried as well as re-swollen freeze-dried and air-dried controls are shown (mean $\pm S D, n=4$ ). 
caffeine $^{25}$ and procaine hydrochloride ${ }^{26}$ for local administrations as well as doxorubicin ${ }^{27}$ or paracetamol $^{28,29}$ for oral delivery. The use of all these drugs delivered by BNC was described earlier. ${ }^{30}$ Since the development of the shapememorized BNC was focused on the use for drug delivery, glucose was selected as a representative additive not only because of its high re-swelling effect but also because of its biocompatibility, established and safe use in many pharmaceutical applications as well as compatibility with the model drug. Loading was performed before air-drying under shaking according to an already established post synthesis loading technique based on diffusional and swelling processes. ${ }^{11}$ All BNC samples were found to take up an equal amount of dye $(12.5 \%$ to $13.5 \%)$ even in the presence of glucose indicating that this process was not influenced by the presence of the additive.

The cumulative release profiles indicated a time-dependent biphasic release profile for the three types of BNC which was represented by an exponential curve with a rapid release in the initial 1 to $2 \mathrm{~h}$ that was followed by a slower release rate up to $168 \mathrm{~h}$ (Fig. 8).

Differences were seen in velocity and extent of dye release depending on the type of BNC that was used as matrix. Nonswellable air-dried controls showed an immediate release of $98.0 \% \pm 5.9 \%$ within 1 hour that was mainly driven by dissolution and diffusion of the dye from the collapsed wetted films along a high concentration gradient between sample and release medium over short distances. The addition of glucose to BNC followed by air-drying retarded the dye release $(52.2 \% \pm$ $2.3 \%$ in $1 \mathrm{~h}$ ) with a total release of $94.9 \% \pm 2 \%$ after $4 \mathrm{~h}$ (airdried glucose). This effect was suggested to rely on the overlay of diffusion and time-dependent swelling being accompanied by an increase of diffusion paths and sample volume. For glucose itself release profiles comparable to azorubine were obtained, with about $64 \%$ during the first hour and $78 \%$ after 4 hours (ESI $4 \dagger$ ). Additionally, glucose might induce a higher water influx into the samples due to its osmotic effect resulting in a faster release compared to the never-dried control. Consequently, the never-dried control that was characterized by the

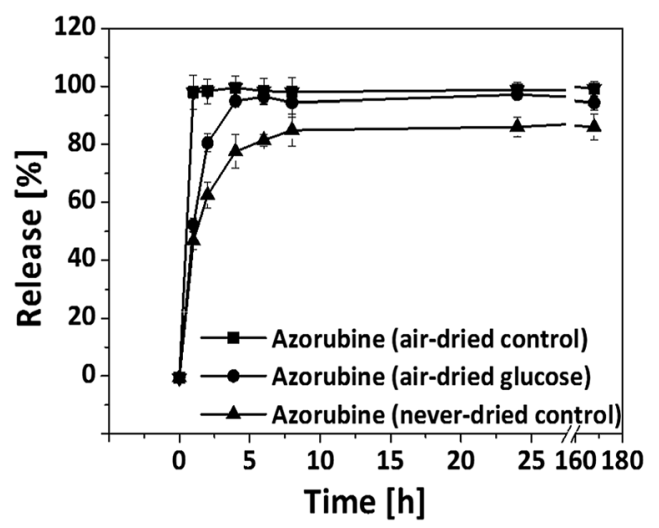

Fig. 8 Time dependency of azorubine release of glucose loaded BNC (air-dried glucose) compared to air-dried and never-dried controls. Azorubine quantification was carried out at $515 \mathrm{~nm}$ spectrophotometrically. Data were calculated as mean \pm SD $(n=3)$. highest hydration, largest sample volume and low swelling, demonstrated the lowest azorubine release.

A second release step $168 \mathrm{~h}$ after the first release was performed by exchange of the release medium to evaluate whether additional azorubine can be released from the samples (data not shown). Longer incubation times up to $120 \mathrm{~h}$ did not considerably increase the removal of the dye (maximal 5.6 to $6.7 \%$ ) for all types of BNC confirming that most of the loaded dye was already released after the first 168 hours.

SEM micrographs of BNC after the release of azorubine and glucose showed an open porous structure (ESI $5 \dagger$ ) with a morphology comparable to re-swollen samples loaded only with glucose (Fig. 6g-i). This confirmed that the porous structure still remains after release of the model drug.

\section{Discussion}

The unique properties of BNC provide the basis for a wide range of applications in the wet and the dried status of this emerging bionanomaterial. However, the conversion of BNC into xerogels is still a major challenge since thermal, mechanical or solvent treatments may change the three-dimensional network structure correlated with a loss of the favorable properties. Air-drying is a cheap, easy-to-perform and solvent-free technique to produce dried BNC, but usually results in a complete collapse of the network forming films with strongly decreased swellability. ${ }^{18}$ Therefore in the present study, several water-binding, hydrophilic additives were identified to produce BNC xerogels with a shape memory effect demonstrating re-swelling, preservation of the native network structure and the mechanical characteristics even after complete air-drying.

Shape-memorized materials were defined as a class of stimuli-responsive materials that are able to retain their original shapes when exposed to an external stimulus. ${ }^{31,32}$ The newly developed material represents a water responsive material capable of gaining its original shape simply by immersing in water by the use of moisture as stimulus. This water-driven shape memory is well known and already described for e.g. polyurethane polymers. ${ }^{3,34}$ The term "shape-memorized" was selected for the additive loaded BNC according to previous reports $^{31,32,35}$ and was introduced earlier for such types of BNC by Numata et al. ${ }^{35}$ who demonstrated a temperature-dependent shape memory effect of BNC after air-drying by the addition of poly(ethylene glycol) of different molecular weights.

Although the selected additives were characterized by different chemical structures, they all are biocompatible, nonvolatile and water-soluble. Some of them were widely described as cryo-as well as lyoprotectants for freeze-drying. ${ }^{36}$

Never-dried BNC fleeces were produced by a highly controlled, standardized and reproducible static cultivation technique as described before. ${ }^{8}$ The shape memory effect was accomplished by post synthesis modification comprising a $24 \mathrm{~h}$ incubation of native, wet BNC in a solution of hydrophilic excipients and a subsequent thorough air-drying to constant mass at room temperature. Based on previous investigations, ${ }^{\mathbf{1 1}}$ the supplementation of BNC with additives was based on the sorption of the excipients mainly driven by diffusion and 
capillary forces induced by the high hydrophilicity and surface area of the BNC nanofiber network equipped with pores and tunnels which were suggested to be responsible for the rapid uptake of agents. ${ }^{37}$ The additives filled the pores of the network leading to a water exchange in the network by the dissolved additives as seen by the increase of the BNC weight after the loading step. Higher molar masses of the additives and their ability (i) to immobilize additional water due to their hydrophilic nature, (ii) to form hydrogen-bonds with the cellulose units and water molecules as well as their (iii) hygroscopicity, might be the reasons for this effect.

The incorporation, crystallization or formation of amorphous glasses of the hydrophilic additives in the BNC network seem to inhibit the crosslinking within the fibre microstructure or formation of lactone bridges that were taken into consideration in the literature for the failure of cellulosic materials to reopen their pores upon contact with water after drying. ${ }^{38}$

Also additives may facilitate the uptake of water during rehydration by active adsorption of water. Their subsequent dissolution forms a high osmotic and concentration gradient between the gel and the surrounding that supports the further influx of water. The varying behavior of different additives was caused by differences in solubility, osmotic activity and hygroscopicity, amorphous glass forming characteristics as well as their different chemical structures and molar masses.

As mentioned before a fast re-swelling comparable to freezedried BNC and a nearly complete micro- and macroscopic reversibility of the BNC network structure to a larger extent than freeze-dried BNC could be achieved by supplementation of BNC with magnesium chloride. The hygroscopic effect of magnesium chloride was shown in several hydrogel applications and was also represented by the high residual water content in the air-dried BNC. For example, polyacrylamide/magnesium chloride hybrid gels demonstrated an increase in swelling with higher magnesium chloride mass and reached the highest swelling degree of $172 \%$ with $85 \%$ magnesium chloride mass. ${ }^{39}$ The magnesium chloride treated, air-dried BNC consisted of $11.1 \mathrm{mg}$ nanocellulose, $54.4 \mathrm{mg}$ additive and $103.4 \mathrm{mg}$ water. Taking into consideration that magnesium chloride (molar mass water-free $95.2 \mathrm{~g} \mathrm{~mol}^{-1}$ ) forms a hexahydrate (molar mass $203.3 \mathrm{~g} \mathrm{~mol}^{-1}$ ), about $61.8 \mathrm{mg}$ of the water content may be related to the hexahydrate formation. For the residual water, it has been described, that magnesium chloride reveals a high water retention even when the relative humidity is below $1 \%{ }^{\mathbf{4 0 , 4 1}}$ and an increasing uptake of water even when the relative humidity is below $33 \%{ }^{\mathbf{4 0 , 4 2 , 4 3}}$ However, the fact that magnesium chloride favors its hexahydrate form as it is thermodynamically stable $^{\mathbf{4 4}}$ was suggested to be not the only reason to explain this behavior. Also the formation of shells and metastable hydrates could be a possible explanation. ${ }^{\mathbf{4 0 , 4 3}}$ The reason for high water retention was described by different groups, ${ }^{45-48}$ who suggested the formation of solid shell structures due to nucleation of solid at the substrate-air-liquid-interface during evaporation, trapping fluids and therefore causing water retention. The low performance of sodium chloride in comparison to magnesium chloride as re-swelling agent can be explained by the strong hygroscopicity of magnesium chloride due to the facilitated hydration of the latter. ${ }^{49}$

In the case of carbohydrates, several parameters such as hygroscopicity and the formation of amorphous glasses during drying have to be taken into consideration. ${ }^{50,51}$ Donnelly et al. ${ }^{\mathbf{5 2}}$ reported hygroscopic properties of monosaccharides and disaccharides at $90 \%$ relative humidity. They found a higher hygroscopicity for glucose and sucrose compared to lactose, which correlates well with the observed re-swelling capacity of carbohydrate supplemented BNC. In contrast, the lower reswelling effect of trehalose correlates with the description of its stability against high relative humidity without any hygroscopicity below 90\% relative humidity. ${ }^{53}$ Therefore, trehalose was used in pharmaceutical and food applications to decrease moisture sensitivity and product caking. ${ }^{54,55}$ Also, lactose $(\alpha$ lactose monohydrate) was shown to be less hygroscopic with absorbing less than $1 \%$ moisture during storage at $\leq 75 \%$ relative humidity. ${ }^{56}$ This correlates well with our results.

Mannitol and sorbitol demonstrated a totally different behavior in the re-swelling experiments, with sorbitol being more effective than mannitol. Although mannitol is an isomer of sorbitol, due to the difference between the two polyols occurring in the planar orientation of the OH-group on the second carbon atom, mannitol resists moisture adsorption even at high relative humidities whereas sorbitol is highly hygroscopic. ${ }^{57}$ Due to this feature sorbitol has been commonly used as plasticizer in hydrogels. ${ }^{\mathbf{5 8 9}}$ Additionally, as described in the literature after air-drying, a strong tendency of mannitol to recrystallize $^{60}$ on top of the BNC fleece surface could be observed that might be an explanation for the lower additive amount in the BNC gels of mannitol compared to sorbitol despite they showed nearly the same water content. The same water content of the isomers could also be due to the fact that under the chosen drying conditions the water adsorption isotherms of both substances were shown to be only slightly different, ${ }^{61}$ suggesting that the hygroscopic effect of sorbitol has only a marginal influence on water content of the dried material, but a strong influence on re-swelling after immersion of the dried samples in the re-swelling medium.

The hygroscopicity of PEG could be ascribed to the presence of ether oxygen atoms in the oxyethylene polymer backbone as well as the hydroxyl end groups, both of which can form numerous hydrogen bonds with water. ${ }^{62}$ The moisturizing effect of PEG was shown in many hydrogel applications such as 2hydroxy-ethyl methacrylate and poly( $N$-[3-(dimethylaminopropyl)]-methacrylamide-co-acrylamide) hydrogels ${ }^{63,64}$ and found to be highly dependent on the degree of polymerization. List and Thron ${ }^{65}$ reported that incubation of $200 \mathrm{Da}$ and 6000 Da PEG in an atmosphere of $65 \%$ relative humidity up to equilibration resulted in a weight increase of $24 \%$ and $1 \%$ for 200 and 6000 Da PEG, respectively. This is in accordance to Baird et al. ${ }^{62}$ who discussed a decreased aqueous solubility for PEG with higher molar masses related to the decrease of the relative fraction of hydroxyl endgroups. Furthermore, Numata et al. ${ }^{35}$ described the preparation of BNC gels using PEG with lower molar masses, showing shape-memory properties of samples swollen by a mixture of 200 Da and 1000 Da PEG. The 
moderate re-swelling effect of the used $8 \mathrm{kDa}$ PEG could therefore be explained by (i) its higher molar mass leading to a lower hygroscopicity and diffusion in the gels, (ii) and an inhibition of water influx into gels due to the high viscosity of this PEG. ${ }^{63}$

Taken these aspects together, the extent and velocity of water uptake and the re-swelling characteristics can be controlled by the selection of an adequate additive. This is also true for the use of the dried BNC as drug delivery system as shown here by the model drug azorubine. Controlling the type of drug release such as simple dissolution, diffusion, swelling or an overlay of different mechanisms may accomplish modified BNC materials with fast or time-resolved and retarded drug release. The selection of the additive has to be adapted and optimized to the requirements of each desired application.

\section{Conclusion}

Although BNC is a material with exceptional characteristics well suited for the application as drug delivery system, it suffers from a poor re-swelling behavior after simple air-drying, making so far time consuming and cost intensive drying techniques necessary. The post modification of BNC with hydrophilic additives is shown here as an easy to perform technique to overcome this issue. Indeed, selected additives like magnesium chloride, glucose and sucrose are able to accomplish a fast water uptake into the dried gels forming hydrogels with nearly unmodified three-dimensional structures and mechanical characteristics.

Easy storage in a dried, stable, space-saving form or a facilitated insertion into cavities of the body with subsequent reswelling to fill the cavity might be special applications for this material. Therefore, the newly developed technique offers the possibility to use these loaded BNC materials not only as drug delivery systems with controllable drug release, but also in biomedical and technical applications as well as in cosmetics and as food additives. For larger BNC dimensions as typically applied in medical applications, the transferability of the process has to be investigated in detail and an up-scaling of the existing process has to be conducted with further optimization. Preliminary tests with larger fleeces already provided first indications that the technique is scalable and applicable for larger and thicker fleeces.

\section{Acknowledgements}

The authors would like to thank Ramona Brabetz and Elena Pfaff for their excellent technical assistance.

\section{Notes and references}

1 D. Klemm, D. Schumann, F. Kramer, N. Hessler, M. Hornung, H.-P. Schmauder and S. Marsch, Adv. Polym. Sci., 2006, 205, 49-96.

2 S. Berndt, F. Wesarg, C. Wiegand, D. Kralisch and F. A. Müller, Cellulose, 2013, 20, 771-783.
3 W. K. Czaja, D. J. Young, M. Kawecki and R. M. J. Brown, Biomacromolecules, 2007, 8, 1-12.

4 A. Svensson, E. Nicklasson, T. Harrah, B. Panilaitis, D. L. Kaplan, M. Brittberg and P. Gatenholm, Biomaterials, 2005, 26, 419-431.

5 N. Bhattarai, J. Gunn and M. Zhang, Adv. Drug Delivery Rev., 2010, 62, 83-99.

6 G. Helenius, H. Baeckdahl, A. Bodin, U. Nannmark, P. Gatenholm and B. Risberg, J. Biomed. Mater. Res., Part A, 2006, 76, 431-438.

7 N. Hessler and D. Klemm, Cellulose, 2009, 16, 899-910.

8 D. Kralisch, N. Hessler, D. Klemm, R. Erdmann and W. Schmidt, Biotechnol. Bioeng., 2010, 105, 740-747.

9 F. Wesarg, F. Schlott, J. Grabow, H.-D. Kurland, N. Hessler, D. Kralisch and F. A. Müller, Langmuir, 2012, 28, 1351813525.

10 D. Klemm, B. Heublein, H.-P. Fink and A. Bohn, Angew. Chem., Int. Ed., 2005, 44, 3358-3393.

11 A. Müller, Z. Ni, N. Hessler, F. Wesarg, F. A. Müller, D. Kralisch and D. Fischer, J. Pharm. Sci., 2013, 102, 579-592.

12 F. Liebner, E. Haimer, M. Wendland, M.-A. Neouze, K. Schlufter, P. Miethe, T. Heinze, A. Potthast and T. Rosenau, Macromol. Biosci., 2010, 10, 349-352.

13 H. Maeda, M. Nakajima, T. Hagiwara, T. Sawaguchi and S. Yano, Kobunshi Ronbunshu, 2006, 63, 135-137.

14 S. Yamanaka, K. Watanabe, N. Kitamura, M. Iguchi, S. Mitsuhashi, Y. Nishi and M. Uryu, J. Mater. Sci., 1989, 24, 3141-3145.

15 N. Hessler, Diploma, Friedrich-Schiller-University Jena, 2004.

16 F. Kramer, D. Klemm, D. Schumann, N. Heßler, F. Wesarg, W. Fried and D. Stadermann, Macromol. Symp., 2006, 244, 136-148.

17 D. Klemm, F. Kramer, S. Moritz, T. Lindström, M. Ankerfors, D. Gray and A. Dorris, Angew. Chem., Int. Ed., 2011, 50, 54385466.

18 C. Clasen, B. Sultanova, T. Wilhelms, P. Heisig and W. M. Kulicke, Macromol. Symp., 2006, 244, 48-58.

19 M. Ul-Islam, W. A. Khattak, M. Kang, S. M. Kim, T. Khan and J. K. Park, Cellulose, 2013, 20, 253-263.

20 K. Fischer, Angew. Chem., 1935, 48, 394-396.

21 H. Fehling, Justus Liebigs Ann. Chem., 1849, 72, 106-113.

22 F. Wesarg, N. Hessler, F. A. Müller, W. Fried, D. Kralisch and

D. Klemm, Conference Proceedings, 239th ACS National Meeting, San Francisco, CA, United States, 2010.

23 D. Klemm, D. Schumann, U. Udhardt and S. Marsch, Prog. Polym. Sci., 2001, 26, 1561-1603.

24 S. Moritz, C. Wiegand, F. Wesarg, N. Hessler, F. A. Mueller, D. Kralisch, U.-C. Hipler and D. Fischer, Int. J. Pharm., 2014, 471, 45-55.

25 N. Lavoine, I. Desloges and J. Bras, Carbohydr. Polym., 2014, 103, 528-537.

26 S. P. Akhlaghi, R. C. Berry and K. C. Tam, Cellulose, 2013, 20, 1747-1764.

27 J. K. Jackson, K. Letchford, B. Z. Wasserman, L. Ye, W. Y. Hamad and H. M. Burt, Int. J. Nanomed., 2011, 6, 321-330. 
28 R. Kolakovic, L. Peltonen, T. Laaksonen, K. Putkisto, A. Laukkanen and J. Hirvonen, AAPS PharmSciTech, 2011, 12, 1366-1373.

29 M. C. I. M. Amin, A. G. Abadi, N. Ahmad, H. Katas and J. A. Jamal, Sains Malays., 2012, 41, 561-568.

30 N. Lin and A. Dufresne, Eur. Polym. J., 2014, 59, 302-325.

$31 \mathrm{X} . \mathrm{Gu}$ and P. T. Mather, RSC Adv., 2013, 3, 15783-15791.

32 J. Jagur-Grodzinski, Polym. Adv. Technol., 2010, 21, 27-47.

33 W. M. Huang, B. Yang, L. An, C. Li and Y. S. Chan, Appl. Phys. Lett., 2005, 86, 114105.

34 J. Mendez, P. K. Annamalai, S. J. Eichhorn, R. Rusli, S. J. Rowan, E. J. Foster and C. Weder, Macromolecules, 2011, 44, 6827-6835.

35 Y. Numata, K. Muromoto, H. Furukawa, J. P. Gong, K. Tajima and M. Munekata, Polym. J., 2009, 41, 524-525.

36 W. Abdelwahed, G. Degobert, S. Stainmesse and H. Fessi, Adv. Drug Delivery Rev., 2006, 58, 1688-1713.

37 D. G. White and R. M. J. Brown, in Cellulose and woodchemistry and technology, ed. C. Schuerch, John Wiley and Sons, Inc, New York, 1989, pp. 573-590.

38 J. M. B. Fernandes Diniz, M. H. Gil and J. A. A. M. Castro, Wood Sci. Technol., 2004, 37, 489-494.

39 Y. Wang and L. Fan, Adv. Mater. Res., 2012, 550-553, 904907.

40 D. J. Cziczo and J. P. D. Abbatt, J. Phys. Chem. A, 2000, 104, 2038-2047.

41 E. Schindelholz, L.-k. Tsui and R. G. Kelly, J. Phys. Chem. A, 2014, 118, 167-177.

42 A. F. Davila, L. G. Duport, R. Melchiorri, J. Jaenchen, S. Valea, A. de los Rios, A. G. Fairen, D. Moehlmann, C. P. McKay, C. Ascaso and J. Wierzchos, Astrobiology, 2010, 10, 617-628. 43 P. Winkler, J. Aerosol Sci., 1973, 4, 373-387.

44 K. Sugimoto, R. E. Dinnebier and J. C. Hanson, Acta Crystallogr., Sect. B: Struct. Sci., 2007, 63, 235-242.

45 V. G. Ciobanu, C. Marcolli, U. K. Krieger, A. Zuend and T. Peter, J. Phys. Chem. A, 2010, 114, 9486-9495.

46 C. Braun and U. K. Krieger, Opt. Express, 2001, 8, 314-321.
47 N. Shahidzadeh-Bonn, S. Rafai, D. Bonn and G. Wegdam, Langmuir, 2008, 24, 8599-8605.

48 C. A. Colberg, U. K. Krieger and T. Peter, J. Phys. Chem. A, 2004, 108, 2700-2709.

49 V. Sudha, S. Harinipriya and M. V. Sangaranarayanan, Chem. Phys., 2005, 310, 59-66.

50 S. Kadoya, K. Fujii, K.-i. Izutsu, E. Yonemochi, K. Terada, C. Yomota and T. Kawanishi, Int. J. Pharm., 2010, 389, 107113.

51 R. H. M. Hatley and J. A. Blair, J. Mol. Catal. B: Enzym., 1999, 7, 11-19.

52 B. J. Donnelly, J. C. Fruin and B. L. Scallet, Cereal Chem., 1973, 50, 512-519.

53 N. K. Jain and I. Roy, Protein Sci., 2009, 18, 24-36.

54 A. Patist and H. Zoerb, Colloids Surf., B, 2005, 40, 107-113.

55 C. Schiraldi, I. Di Lernia and M. De Rosa, Trends Biotechnol., 2002, 20, 420-425.

56 Y. Listiohadi, J. A. Hourigan, R. W. Sleigh and R. J. Steele, Int. J. Pharm., 2008, 359, 123-134.

57 S. Yoshioka, Y. Aso and T. Kawanishi, Pharmacuetical Regul. Sci., 2008, 39, 51-60.

58 A. Nishida, M. Yamada, T. Kanazawa, Y. Takashima, K. Ouchi and H. Okada, Chem. Pharm. Bull., 2010, 58, 1480-1486.

59 S.-I. Hong, J.-W. Lee and S.-M. Son, Packag. Technol. Sci., 2005, 18, 1-9.

60 L. Yu, D. S. Mishra and D. R. Rigsbee, J. Pharm. Sci., 1998, 87, 774-777.

61 M. Fischer and G. Schepky, Drug Dev. Ind. Pharm., 1995, 21, 279-300.

62 J. A. Baird, R. Olayo-Valles, C. Rinaldi and L. S. Taylor, J. Pharm. Sci., 2009, 99, 154-168.

63 A. K. Bajpai and M. Shrivastava, J. Biomater. Sci., Polym. Ed., 2002, 13, 237-256.

64 G. B. Demirel, T. Caykara, M. Demiray and M. Guru, J. Macromol. Sci., Part A: Pure Appl. Chem., 2009, 46, 58-64.

65 P. H. List and T. Thron, Indian J. Pharm., 1978, 40, 253-256. 